\title{
Development of Large-Area Charged Particle Detector with Inorganic Scintillator Plates and Wavelength Shifting Fibers
}

\author{
Takahiro MIZUNO, Yusaku EMOTO, Kento FUJIHARA, Hiroshi ITO, Hideyuki KAWAI, Shota KIMURA, and \\ Atsushi KOBAYASHI
}

Email: takahiro.hqa125@gmail.com

\begin{abstract}
A Large-area charged particle detector for high energy physics experiments has been developed. This detector includes inorganic scintillator plates with thicknesses of $0.5 \mathrm{~mm}$ and wavelength-shifting fibers (WLSFs) of $0.2 \mathrm{~mm}$ in diameters. The size of effective area is $1 \mathrm{~m}$ by $1 \mathrm{~m}$. The WLSFs are connected to both plate surfaces optically, and on the top and the bottom, fibers are attached along to $x$ and $y$-axis direction, respectively. The best WLSF to obtain large number of photoelectrons is determined, which enables us to detect charged particles with thinner scintillation crystals. This means an improvement of position resolution of this detector. The number of photoelectrons obtained from a new type scintillation crystal, which is more reasonable than the conventional ones are also measured. We also estimated its position resolution. This detector enables us to detect charged particles with higher position resolution and lower cost than conventional scintillation detectors and gas chambers.
\end{abstract}

\section{INTRODUCTION}

When charged particles pass thorough a scintillation crystal, scintillation light is released isotropically and the light with smaller incident angle than the critical angle goes outside the crystal. The area of the light emission region on the crystal depends on the location of the light emitting point and the thickness of the crystal. As shown in Fig. 1, the emission region gets smaller as the crystal thinner. To curb this spread of the light, inorganic crystals with high densities are introduced.
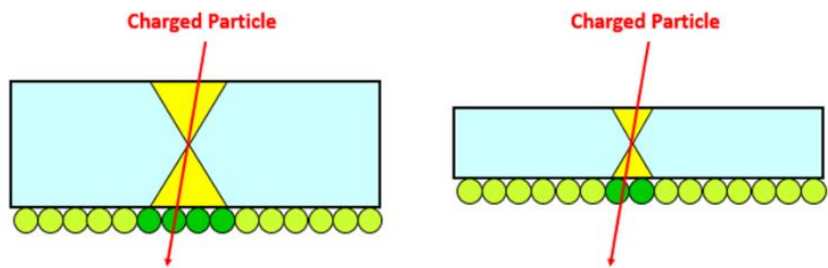

Fig. 1. Using a high density and thin inorganic crystal enables us to detect charged particles with high position resolution. When the crystal is thin, the distance to the boundary surface is short, and the spread of the light is smaller.

As a scintillation crystal, La-GPS(Ce) scintillator is used. It has chemical composition of $\left(\mathrm{La}_{0.75} \mathrm{Ga}_{0.24} \mathrm{Ce}_{0.01}\right)_{2} \mathrm{Si}_{2} \mathrm{O}_{7}$. It emits 30,000 to 40,000 photons $/ \mathrm{MeV}$. The emission wavelength is 390 to $410 \mathrm{~nm}$. Its density is $5.3 \mathrm{~g} / \mathrm{cm}^{3[1]}$. Wavelength-shifting fiber (WLSF) is a kind of optical fiber. It absorbs the light of the particular wavelength and emits longer wavelength light. WLSF has approximately $1 \mathrm{~m}$ of attenuation length. Fibers are arranged on the crystals and lead the light to the photodetectors. As shown in Fig. 2, charged particles are detected with several layers of WLSFs on the crystal. They are arranged both longitudinally and laterally, so the lightemitting point is determined. One of the WLSF layers is composed of 5000 fibers of $0.2 \mathrm{~mm}$ in diameter.

\section{Charged Particle}

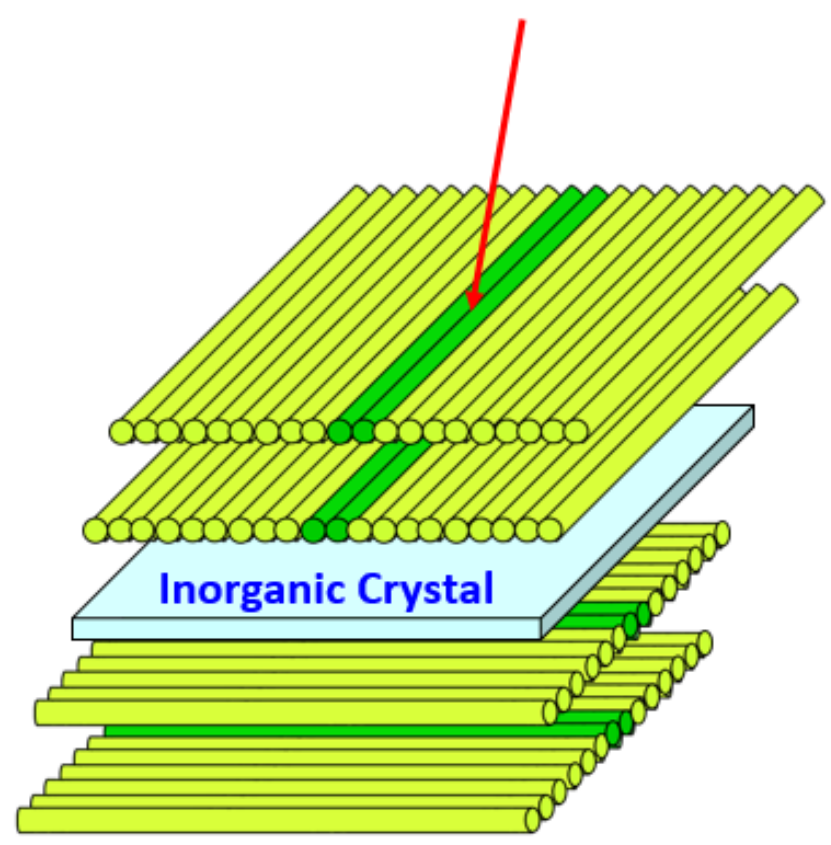

Fig. 2. The detection part is composed of several layers of WLSF on the inorganic scintillation crystal. They are arranged longitudinally and laterally.

We measured the number of photoelectrons with two kinds of fibers B-3 (300) MJ and Y-11 (300) MJ, which are manufactured by Kuraray Co. Ltd. to determine which kind of WLSF is the best to read out the light. We also measured the number of photoelectrons with high-growth-rate La-GPS crystal. High-growth-rate scintillation crystal is a scintillator which is crystalized at the higher rate than the conventional one. It is much more reasonable than the conventional one while it contains micro bubble and is not transparent completely. 


\section{EXPERIMENTAL}

The number of photoelectrons was measured by using the La-GPS scintillation crystals. As a WLSF, B-3 (300) MJ and Y-11 (300) MJ are used. Fig. 3. shows the La-GPS crystal and the WLSFs used in this experiment.
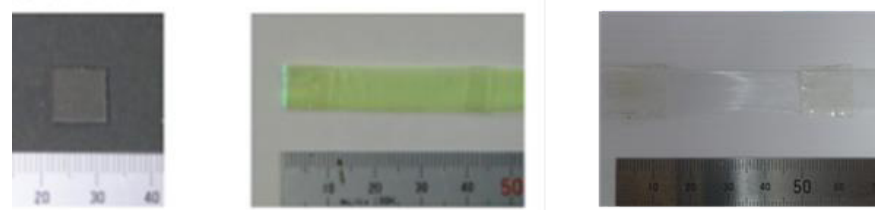

Fig. 3. The La-GPS crystal (left) with the thickness of $0.50 \mathrm{~mm}$ is used as a scintillator. The Y-11 (300) MJ WLSFs (center) and the B-3 (300) MJ WLSFs (right) are $0.2 \mathrm{~mm}$ in diameter. They are arranged in sheet-like.

La-GPS crystal is manufactured by C\&A Co., Ltd., which has the thickness of $0.50 \mathrm{~mm}$ and yield 30000 to 40000 photons/MeV light output. Peak wavelength of emission light is 390 to $410 \mathrm{~nm}^{[1]}$. B-3 (300) MJ and Y-11 (300) MJ WLSFs are made by Kuraray Co. Ltd., which are $0.2 \mathrm{~mm}$ in diameters. B-3 (300) MJ Fiber has emission peak of $450 \mathrm{~nm}$ and absorption peak of $351 \mathrm{~nm}^{[2]}$. Y-11 (300) MJ Fiber has emission peak of $476 \mathrm{~nm}$ and absorption peak of $430 \mathrm{~nm}^{[2]}$. Photomultiplier tubes of R9880U-210 series manufactured by Hamamatsu Photonics K.K. are used as the photodetectors. They have ultra bialkali photocathodes of $8 \mathrm{~mm}$ in diameter. They have spectral response in the wavelength range of 230 $\mathrm{nm}$ to $700 \mathrm{~nm}$ and the peak wavelength of response is 400 $\mathrm{nm}^{[3]}$.

The number of photoelectrons was measured by changing the number of layers of WLSFs. Fig. 4. shows the setup of the experiment. The $\beta$-ray from the ${ }^{90} \mathrm{Sr}$ radiation source was detected by the $0.50 \mathrm{~mm}$ La-GPS crystal. The crystal was covered with the WLSF sheet. WLSF sheets with 1 to 6 layers of B-3(300) MJ and WLSF sheets with 1 to 6 layers of Y-11 (300) MJ are prepared. A PMT was attached to the end of the sheet. The detection was triggered by three PMTs. Two bundles of 5 scintillation fibers of $1.0 \mathrm{~mm}$ in diameters were placed orthogonally immediately above the detection part and each bundle was attached to a PMT. Another scintillation crystal was placed immediately below and attached to another PMT.

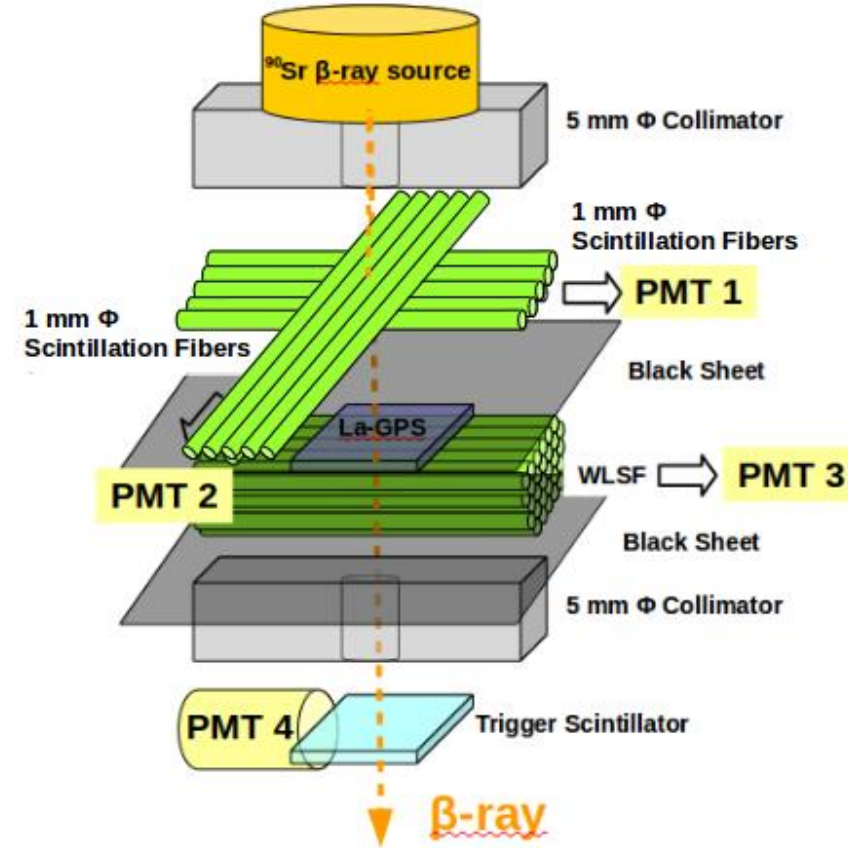

Fig. 4. The setup of the experiment is shown above. The detection is triggered by three PMTs.

The number of photoelectrons using high-growth-rate LaGPS with the thickness of $0.55 \mathrm{~mm}$ was measured in the same set-up. Taking the results of former experiment into consideration, we experimented using WLSF sheets with 1 to 6 layers of B-3 (300) MJ. Fig. 5. shows the high-growth-rate La-GPS crystal used in this experiment.

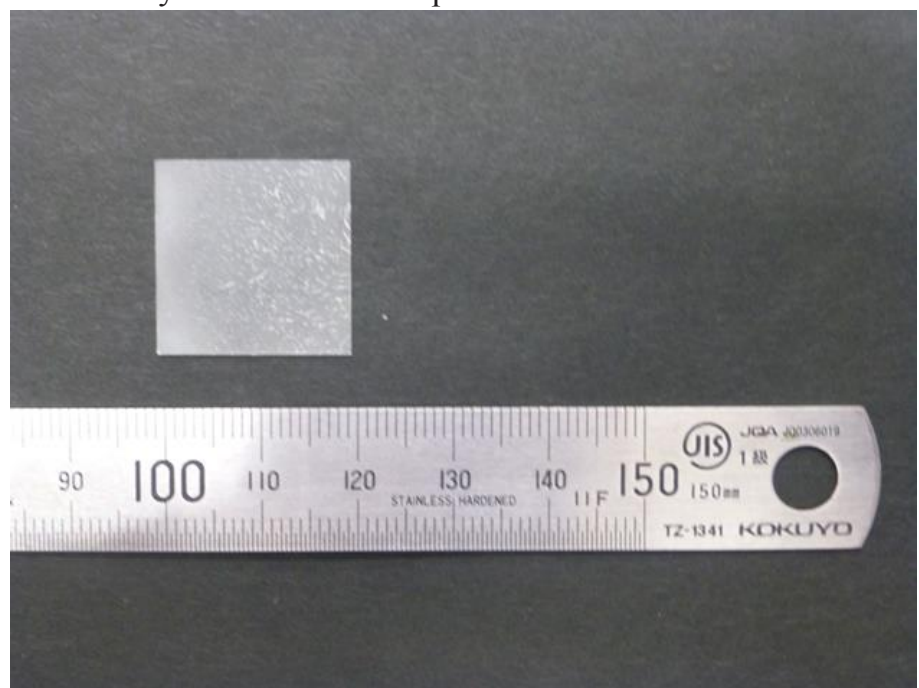

Fig. 5. The high-growth-rate La-GPS crystal with the thickness of $0.55 \mathrm{~mm}$ used in the experiment is shown above. It contains micro bubble and is not transparent completely. However, it is more reasonable than conventional ones. 


\section{RESULTS}

The results for Y-11 (300) MJ WLSF are shown in Fig. 6. and Fig. 7. The number of photoelectrons is a value on one side reading.

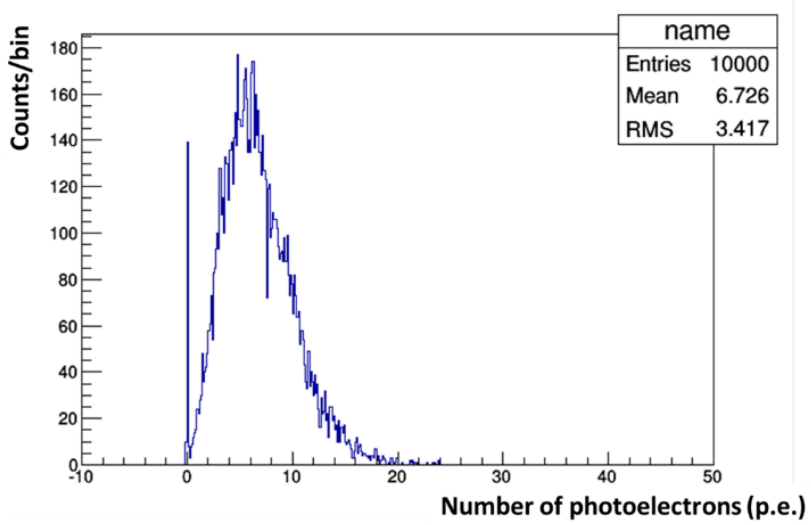

Fig. 6. The distribution of the numbers of photoelectrons detected with $0.50 \mathrm{~mm}$ thick La-GPS crystal, 6 layers Y-11 (300) MJ WLSF on one-side reading is shown above. 6.7 photoelectrons are detected in average.

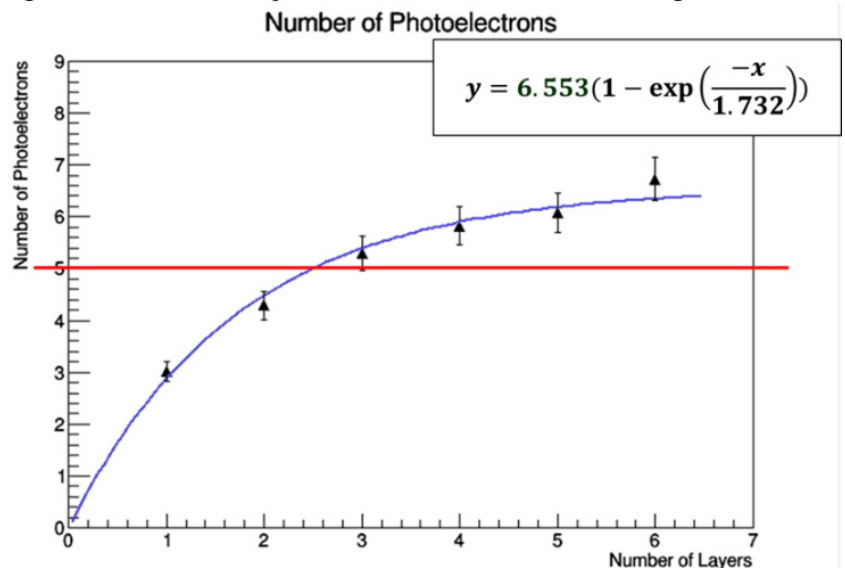

Fig. 7. Averaged numbers of photoelectrons detected on one-side reading for $0.50 \mathrm{~mm}$ La-GPS crystal by changing the number of Y-11 (300) MJ WLSF layers is shown above.

According to Fig. 7., the numbers of photoelectrons are gradually approaching a specific value. The experimental data could be approximated to a function $\mathrm{y}=6.553(1-\mathrm{e}-\mathrm{x} / 1.732)$, and this means the number of photoelectrons is saturated to 6.553 as increasing the number of layers. Energy deposit in $1.0 \mathrm{~mm}$ crystal is approximately $1 \mathrm{MeV}$, thus approximately 13 photoelectrons per $1 \mathrm{MeV}$ can be collected. Considering the number of photoelectrons is doubled with both sides reading, 3 WLSF layers are enough for detecting 10 photoelectrons, which corresponds to of $99 \%$ or more detection efficiency.

The results for B-3 (300) MJ WLSF are shown in Fig. 8. and Fig. 9. The number of photoelectrons is a value on one side reading.

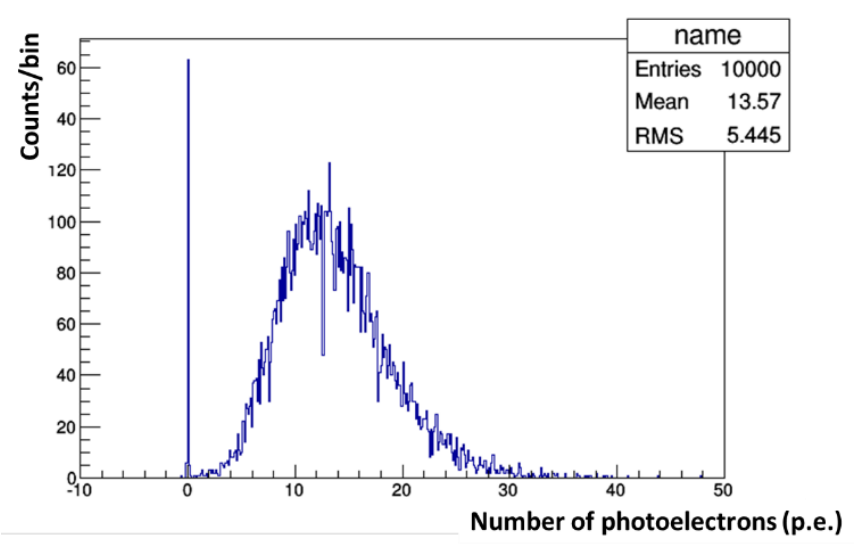

Fig. 8. The distribution of the numbers of photoelectrons detected with $0.50 \mathrm{~mm}$ thick La-GPS crystal, 6 layers B-3 (300) MJ WLSF on one-side reading is shown above. 13.6 photoelectrons are detected in average.

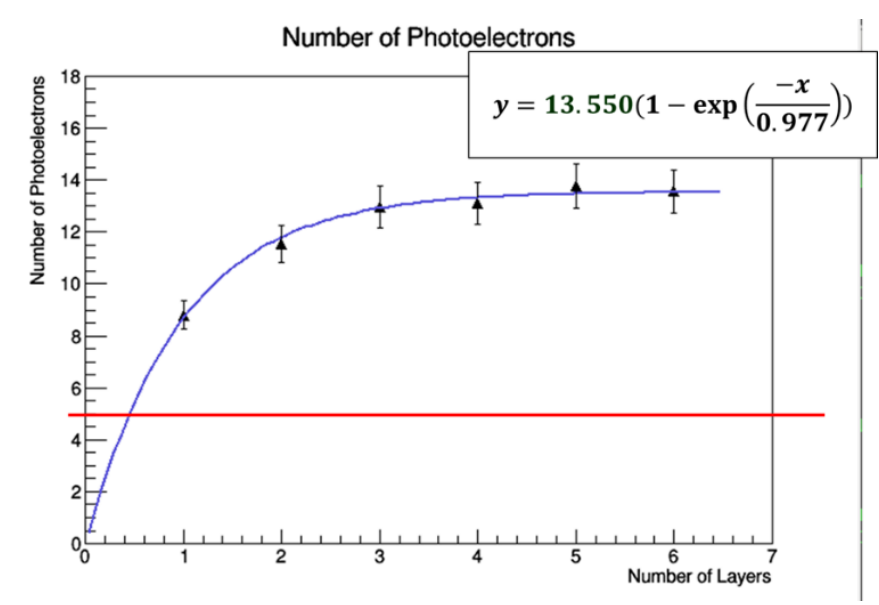

Fig. 9. Averaged numbers of photoelectrons detected on one-side reading for $0.50 \mathrm{~mm}$ La-GPS crystal by changing the number of B-3 (300) MJ WLSF layers is shown above.

According to Fig. 9., the numbers of photoelectrons are gradually approaching a specific value. The experimental data could be approximated to a function $y=13.550(1-\mathrm{e}-\mathrm{x} / 0.977)$, and this means the number of photoelectrons is saturated to 13.550 as increasing the number of layers. Energy deposit in $1.0 \mathrm{~mm}$ crystal is approximately $1 \mathrm{MeV}$, thus approximately 27 photoelectrons per $1 \mathrm{MeV}$ can be collected. Considering the number of photoelectrons is doubled with both sides reading, 1 WLSF layer is enough for detecting 10 photoelectrons, which corresponds to of $99 \%$ or more detection efficiency.

The result for B-3 (300) MJ WLSF and $0.55 \mathrm{~mm}$ thick highgrowth-rate La-GPS is shown in Fig. 10. The number of photoelectrons is a value on one side reading. 


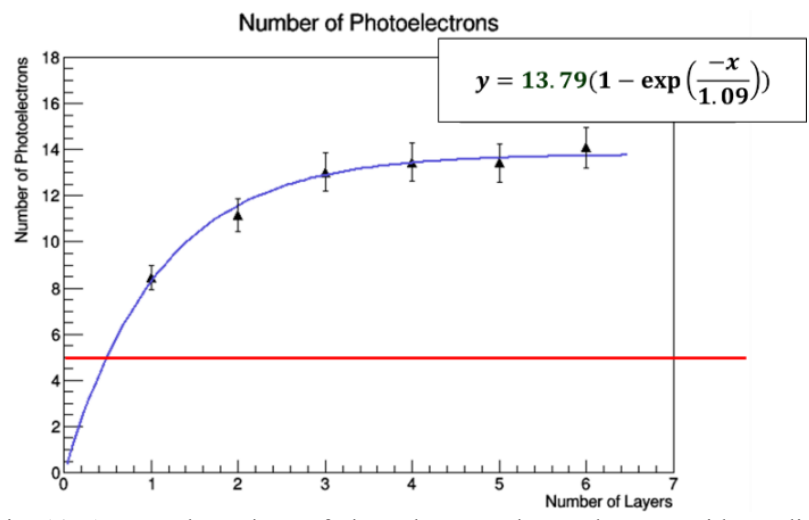

Fig. 10. Averaged numbers of photoelectrons detected on one-side reading for $0.55 \mathrm{~mm}$ thick high-growth-rate La-GPS crystal by changing the number of B-3 (300) MJ WLSF layers is shown above.

According to Fig. 10., the numbers of photoelectrons are gradually approaching a specific value. The experimental data could be approximated to a function $\mathrm{y}=13.79(1-\mathrm{e}-\mathrm{x} / 1.09)$, and this means the number of photoelectrons is saturated to 13.79 as increasing the number of layers. It corresponds to 12.54 photoelectrons for $0.50 \mathrm{~mm}$ high-growth-rate La-GPS. Energy deposit in $1.0 \mathrm{~mm}$ crystal is approximately $1 \mathrm{MeV}$, thus approximately 25 photoelectrons per $1 \mathrm{MeV}$ can be collected. Considering the number of photoelectrons is doubled with both sides reading, 1 WLSF layer is enough for detecting 10 photoelectrons, which corresponds to of $99 \%$ or more detection efficiency. The number of photoelectrons when using high-growth-rate La-GPS is approximately equal to the value when using conventional one.

\section{Estimation OF THE Position Resolution}

The position resolution is estimated using $0.5 \mathrm{~mm}$ thick LaGPS crystal and 1 layer B-3 (300) MJ WLSF sheet which is composed of $10 \mathrm{WLSF}$. The setup of the experiment is shown in Fig. 11.

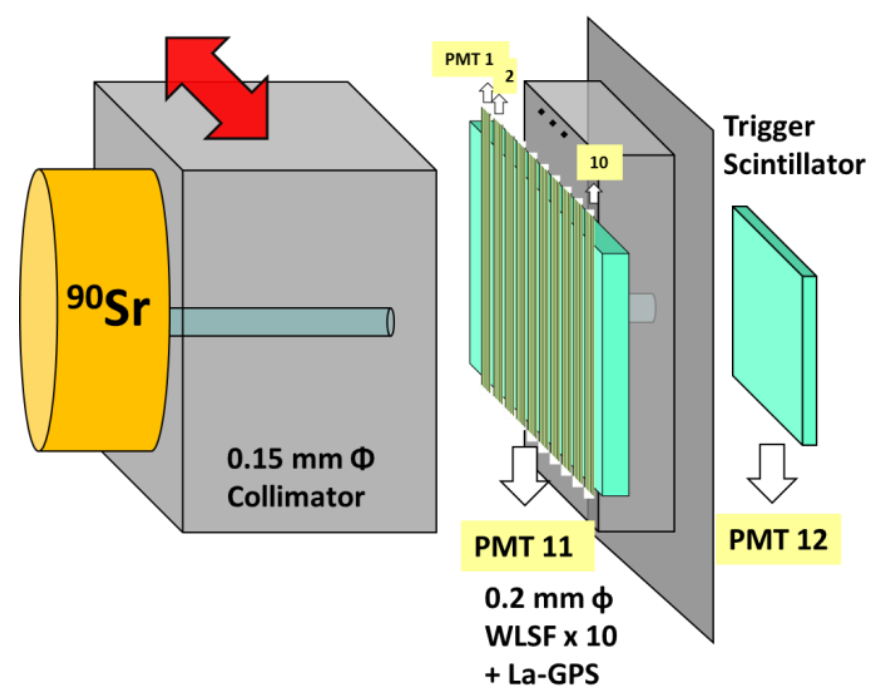

Fig. 11. The setup of the experiment is shown above. The detection is triggered by three PMT 11 and PMT 12.
The number of the photoelectrons is measured by reading out from one end of each WLSF. The position where the charged particle has passed thorough is determined by using the center of gravity calculation with the number of photoelectrons emitted from each WLSF. All 10 WLSFs are bundled and all photoelectrons emitted from fibers are read out at another end of WLSF sheet. The detection is triggered by PMT 11 and PMT 12.

Fig. 12. shows the one result of measurements of position resolution. The sigma of the distribution of reconstruction points is $0.326 \mathrm{~mm}$.

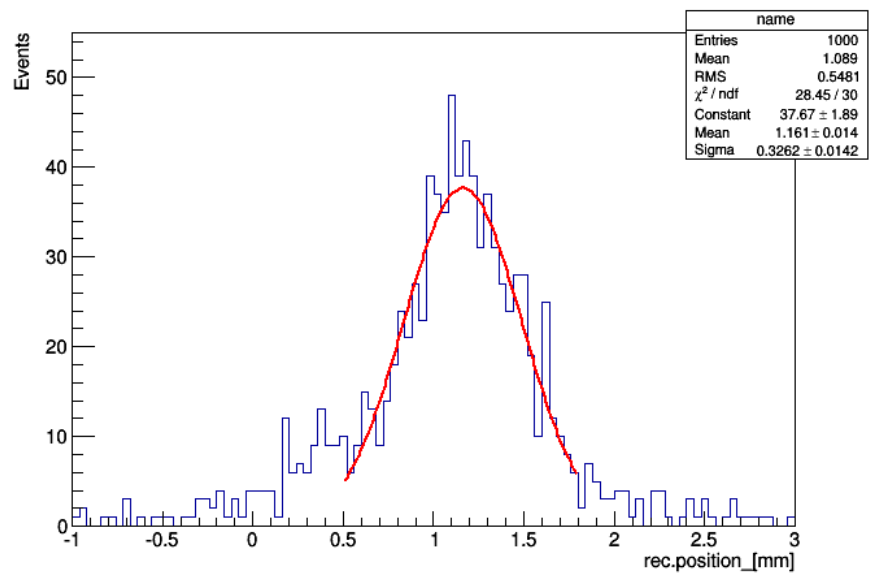

Fig. 12. The result of center of gravity calculation is shown above. The sigma of the distribution of reconstruction points is $0.326 \mathrm{~mm}$.

As a result of 6 times measurements, the position resolution is $0.403 \pm 0.082 \mathrm{~mm}$ in sigma.

\section{DISCUSSION}

We propose the large-area charged particle detector with inorganic scintillator plates and wavelength shifting fibers. It is confirmed that photons from La-GPS can be read out with WLSF B-3 (300) MJ and Y-11 (300) MJ. When using $0.5 \mathrm{~mm}$ thickness La-GPS and B-3 (300) MJ, the more photoelectrons can be obtained and 1 WLSF layer is enough for detecting 10 photoelectrons with both sides reading. The same result is obtained when using high-growth-rate La-GPS. This is better result than 3 layers for $1.0 \mathrm{~mm}$ crystal ${ }^{[4]}$. Its position resolution is $0.403 \pm 0.082 \mathrm{~mm}$ in sigma.

If using 2 layers of WLSF sheets for detection in each direction, only 300 read-out circuits are necessary for each direction; 5000 fibers in each sheet is divided into 50 bundles and each fiber is assigned a number 1 to 100 in each bundle. The fiber assigned to the same number is attached to one PMT. In the first layer, fired bundle is determined with $50 \times 2$ (bothsides reading) PMTs and in the second layer, the fired fiber is determined with $100 \times 2$ (both-sides reading) PMTs.

By using these inorganic crystals and WLSFs, "high position resolution" and "lower cost" detector can be made. In addition, photo detectors are suitable for measurements at high event rate. This detector may take the place of other conventional detectors. 


\section{ACKNOWLEDGMENT}

We are grateful to Assoc. Prof. K. Kamada and Prof. A.

Yoshikawa, Tohoku University for providing scintillation

crystals.

\section{REFERENCES}

[1] C\&A Corporation, Product Information Available: www.c-and-a.jp/GPS.html.

[2] Kuraray, Wavelength Shifting Fibers Available: kuraraypsf.jp/psf/ws.html.

[3] Hamamatsu Photonics K.K., Photomultiplier tube R9880U-210 Available: https://www.hamamatsu.com/jp/en/R9880U-210.html

[4] T. Mizuno et al. "Development of Large-Area Charged Particle Detectors with High Position Resolution and Low Cost", 2006 IEEE Nuclear Science Symposium Conference. 